Pacific Journal of Mathematics

PARTIAL ALGEBRAIC STRUCTURES ASSOCIATED WITH 


\title{
PARTIAL ALGEBRAIC STRUCTURES ASSOCIATED WITH ORTHOMODULAR POSETS
}

\author{
STANLEY P. GUdDER
}

\begin{abstract}
In this paper there are three main results:
I. An orthomodular poset with property $C$ is essentially the same as an associative partial Boolean algebra.

II. If $P$ is an orthomodular poset, then $S(P)$, the set of residuated maps on $P$, can be made into a weak partial Baer*. semigroup is such a way that $P$ is isomorphic to the orthomodular poset of closed projections in $S(P)$.

III. If $(P, M)$ is a conditional quantum logic, then the collection of all finite compositions of primitive operations (satisfying certain technical conditions) is a partial Baer*-semigroup.

It is assumed that the reader is familiar with the rudiments of the theory of orthomodular posets.
\end{abstract}

1. Introduction. Although partial algebraic structures seem to arise in many areas of mathematics, their systematic study seems to have been neglected. Among the few studies of such structures known to the author are the connection between congruence relations and partial algebras $[4,5]$, the coordinatization of partially ordered sets and partial Baer*-semigroups $[6,18]$, and considerations of the "logic" of quantum mechanics $[9,11,12]$. It is the purpose of this paper to consider some partial algebraic structures and to develop at least a small portion of their theory with the hope of stimulating further interest and research in this field.

By a partial algebraic structure we mean a set $S$ with certain "partial" operations in the sense that these operations are defined only for certain elements of $S$. For example let $S$ be an ordinary algebraic structure and let $T \subseteq S$. If we restrict the operations of $S$ to those elements which are in $T$ after the operations are perfomed then we obtain a partial algebraic structure. In particular, let $(S, \cdot)$ be a semigroup and let $T$ be a nonempty subset of $S$. Define a relation $R \subseteq S \times S$ by $(a, b) \in R$ if $a \cdot b \in T$. We then have a map $R \rightarrow S$ given by $(a, b) \rightarrow a \cdot b$ and we call $(S, R, \cdot)$ a partial semigroup (also $(T, R \cap$ $(T \times T), \cdot)$ is a partial semigroup). We say that these partial semigroups are generated by the subset $T$ of the semigroup $S$. Notice that we have a partial associative law in these partial semigroups in the sense that if $(a, b) \in R$ and $(b, c) \in R$ then $(a \cdot b, c) \in R$ if and only if $(a, b \cdot c) \in R$ and in that case $(a \cdot b) \cdot c=a \cdot(b \cdot c)$.

As another example, let $S$ be the set of all rectangular matrices with real entries. Then the natural addition can only be applied to 
matrices of the same size and thus gives a partial binary operation on $S$. Similarly the natural multiplication of two matrices $A$ and $B$ of size $m \times n$ and $r \times s$ respectively can only be applied if $n=r$ and again we obtain a partial binary operation on $S$. In this way $S$ becomes a partial ring. In analysis (and, to a lesser extent, other branches of mathematics) one frequently considers a set $S$ and mappings from $S$ to some set $T$ that have domains which in general are proper subsets of $S$. Meaningful and useful operations between these mappings such as composition can be applied only if their domains satisfy certain criteria. For example in the important study of closed densely defined unbounded linear transformations on Banach spaces if the sum of two such transformations is to be a transformation of the same type one must have that the intersection of their domains is dense. In this way summation becomes a partial binary operation.

2. Partial semigroups and partial Boolean rings. In this section we consider partial semigroups and partial Boolean rings and give their connection to known algebraic systems.

Let $L=\{a, b, c, \cdots\}$ be a semi-lattice. It is well known [1] that if we define $a \cdot b=a \wedge b$ then $(L, \cdot)$ becomes a commutative idempotent semigroup. Conversely if $(S, \cdot)$ is a commutative idempotent semigroup and we define $a \leqq b$ if $a \cdot b=a$, then $(S, \leqq)$ is a semi-lattice. These results can be generalized to posets if partial operations are considered.

Let $S=\{a, b, c, \cdots\}$ be a set and let $R \subseteq S \times S$ be a relation on $S$. We say that $(S, R, \cdot)$ is a partial semigroup if there is a map from $R$ into $S$ denoted by $(a, b) \rightarrow a \cdot b$ with the following property: for any $a, b, c \in S$ such that $(a, b),(b, c) \in R$ then $(a, b \cdot c) \in R$ if and only if $(a \cdot b, c) \in R$ and in that case $(a \cdot b) \cdot c=a \cdot(b \cdot c)$. It is clear that the relation $R$ gives the pairs of elements that can be multiplied and that the above property is a partial associative law. An interesting question that we shall not answer here is whether any partial semigroup is generated by a subset of a semigroup and if not characterize those that are so generated. A partial semigroup $(S, R, \cdot)$ is idempotent if $(a, a) \in R$ and $a \cdot a=a$ for all $a \in S . \quad(S, R, \cdot)$ is commutative if $(a, b) \in$ $R$ implies $(b, a) \in R$ and $b \cdot a=a \cdot b$.

Theorem 2.1. Let $(P, \leqq)$ be a poset. Define a relation $R$ on $P$ by $(a, b) \in R$ if $a \leqq b$ or $b \leqq a$ and a partial binary operation by $a \cdot b=$ $a \wedge b$ if $(a, b) \in R$. Then $(P, R, \cdot)$ is a commutative idempotent partial semigroup.

Proof. Clearly $(a, a) \in R$ and $a \cdot a=a$ for all $a \in P$. If $(a, b) \in R$ then $(b, a) \in R$ and $b \cdot a=a \wedge b=a \cdot b$. Suppose $(a, b) \in R$ and $(b, c) \in$ $R$. We now have four cases: 
1. $a \leqq b, b \leqq c$. Then $a \cdot b=a$ and $b \cdot c=b$ so $(a \cdot b, c) \in R$ and $(a, b \cdot c) \in R$ both hold automatically.

2. $a \leqq b, b \geqq c$. Then $a \cdot b=a$ and $b \cdot c=c$ so $(a \cdot b, c) \in R$ if and only if $(a, b \cdot c) \in R$.

3. $a \geqq b, b \leqq c$. Then $a \cdot b=b$ and $b \cdot c=b$ so $(a \cdot b, c) \in R$ and $(a, b \cdot c) \in R$ both hold automatically.

4. $a \geqq b, b \geqq c$. Then $a \cdot b=b, b \cdot c=c$ so $(a \cdot b, c) \in R$ and $(a, b \cdot c)$ both hold automatically.

Since $(a \cdot b) \cdot c=a \wedge b \wedge c=a \cdot(b \cdot c)$ the proof is complete.

THeOREM 2.2. Let $(S, R, \cdot)$ be a commutative idempotent partial semi-group. Define $a \leqq b$ if $(a, b) \in R$ and $a \cdot b=a$. Then $(S, \leqq)$ is a poset.

Proof. Clearly $a \leqq a$ for all $a \in S$. If $a \leqq b$ and $b \leqq a$, then $a=a \cdot b=b \cdot a=b$. If $a \leqq b$ and $b \leqq c$ then $(a, b) \in R,(b, c) \in R, a \cdot b=$ $a$, and $b \cdot c=b$. Since $(a, b \cdot c) \in R$ we have $(a, c)=(a \cdot b, c) \in R$ and $a=$ $a \cdot b=a \cdot(b \cdot c)=(a \cdot b) \cdot c=a \cdot c$. Hence $a \leqq c$ which completes the proof.

Kochen and Specker [11, 12] define a partial algebraic structure they call a partial Boolean ring which they use in their studies of quantum logics and hidden variable theories in quantum mechanics. A partial Boolean ring $(A, R,+, \cdot, 0,1)$ consists of a set $A=\{a, b, c, \cdots\}$ a binary relation $R$ on $A$ called commeasurability, two partial binary operations + and $\cdot$, and two nullary operations 0 , 1 which satisfy:

1. if $(a, b) \in R$ then $a+b$ and $a \cdot b$ are defined;

2. $R$ is reflextive and symmetric;

3. for every $a \in A,(a, 0),(a, 1) \in R, a+0=a, 1 \cdot a=a$;

4. if $\left(a_{i}, a_{j}\right) \in R, i, j=1,2,3$ then $\left(a_{1}+a_{2}, a_{3}\right) \in R,\left(a_{1} \cdot a_{2}, a_{3}\right) \in R$;

5 . any three mutually commeasurable elements generate a Boolean ring relative to $(+, \cdot, 0,1)$.

A partial Boolean ring $(A, R,+, \cdot, 0,1)$ is associative if $(A, R, \cdot)$ is a partial semigroup. Now let $P$ be an orthomodular poset. That is, $P$ is a poset with first and last elements 0 , 1, an orthocomplementation $a \rightarrow a^{\prime}$ satisfying $a^{\prime \prime}=a, a \leqq b$ implies $b^{\prime} \leqq a^{\prime}, a \vee a^{\prime}=1$ for all $a \in P$, if $a$ and $b$ are disjoint (i.e., $a \leqq b^{\prime}$ ) then $a \vee b$ exists and finally if $a \leqq b$ then $b=a \vee\left(b \wedge a^{\prime}\right)$. If $a \leqq b$ we use the notation $b-a=b \wedge a^{\prime}$. We say that $a, b \in P$ are compatible and write $a C b$ if there exist mutually disjoint elements $a_{1}, b_{1}, c$ such that $a=$ $a_{1} \vee c$ and $b=b_{1} \vee c$. It is clear that $C$ is a reflextive, symmetric relation on $P$. It can be shown $[7,15,16,19]$ that $a, b \in P$ are in a Boolean subalgebra of $P$ if and only if $a C b$. We say that $P$ has property $C$ if whenever $a, b, c$ are mutually compatible we have $(a \vee b) C c$. It can be shown [7, 15] that if $P$ satisfies condition $C$ then a subset $S$ of $P$ is contained in a Boolean subalgebra of $P$ if 
and only if the elements of $S$ are mutually compatible. We now show that there is an intimate connection between partial Boolean rings and orthomodular posets.

THEOREM 2.3. Let $P$ be an orthomodular poset with property $C$. If $a C b$ define $a \cdot b=a \wedge b$ and $a+b=(a-a \wedge b) \vee(b-a \wedge b)$. Then $(P, C,+, \cdot, 0,1)$ is an associative partial Boolean ring.

Proof. The only part of the proof that is not immediate is to show that $(P, C,+, \cdot, 0,1)$ is associative. We must show that if $a C b$ and $b C c$ then $a \cdot b C c$ if and only if $a C b \cdot c$ and in this case $(a \cdot b) \cdot c=$ $a \cdot(b \cdot c)$. However, this follows from [6, Lemma 3.3].

THEOREM 2.4. Let $(A, R,+, \cdot, 0,1)$ be an associative partial Boolean ring. Define $a \leqq b$ if $(a, b) \in R$ and $a \cdot b=a$ and define $a^{\prime}=$ $1-a$. Then $\left(A, \leqq,^{\prime}\right)$ is an orthomodular poset with property $C, R \leqq C$ and if $(a, b) \in R$ then $a \wedge b=a \cdot b$ and $a \vee b=a+b-a \cdot b$. If whenever $a, b$ is contained in a Boolean subring we have $(a, b) \in R$ then $R=C$.

Proof. That $\leqq$ is antisymmetric and reflexive follows from (5). Now suppose $a \leqq b$ and $b \leqq c$. Since $(a, b),(b, c)$ and $(a, b \cdot c) \in R$, applying the associativity of $A$ we have $(a, c)=(a \cdot b, c) \in R$ and $a=$ $a \cdot b=a \cdot(b \cdot c)=(a \cdot b) \cdot c=a \cdot c$ so $a \leqq c$. Thus $(A, \leqq)$ is a poset. It follows from (3) and (5) that $0 \cdot a=0$ and $1 \cdot a=1$ for all $a \in A$ so 0 and 1 are the first and last elements of $A$ respectively. Now $a^{\prime \prime}=$ $1-(1-a)=a$. If $b \geqq a, a^{\prime}$ then $a=a \cdot b$ and $(1-a)=(1-a) \cdot b$ so $1-a=b-a \cdot b=b-a$. Hence $b=1$ and we have $a \vee a^{\prime}=1$ for all $a \in A$. If $a \leqq b$ then $(1-a) \cdot(1-b)=1-b-a+a \cdot b=1-b$ so $b^{\prime} \leqq a^{\prime}$. Hence $\left(A, \leqq,^{\prime}\right)$ is an orthocomplemented poset. If $(a, b) \in R$ then $a \cdot b \leqq a, b$ by (2), (4) and (5). Now suppose $c \leqq a, b$. Then $a, b, c$ are mutually commeasurable and hence by $(4),(c, a \cdot b) \in R$ and by (5) $c \cdot(a \cdot b)=(c \cdot a) \cdot b=c \cdot b=c$ so $c \leqq a \cdot b$. Hence a $\wedge b=a \cdot b$. Also if $(a, b) \in R$ then $\left(a^{\prime}, b^{\prime}\right) \in R$ and $a \vee b=\left(a^{\prime} \wedge b^{\prime}\right)^{\prime}=1-(1-a) \cdot(1-b)=$ $a+b-a \cdot b$. To show $\left(A, \leqq,^{\prime}\right)$ is orthomodular suppose $a \leqq b$. Then $(a, b) \in R \quad$ so $\quad a \vee\left(b \wedge a^{\prime}\right)$ exists and $a \vee\left(b \wedge a^{\prime}\right)=a+$ $\left(b \wedge a^{\prime}\right)-a \cdot\left(b \wedge a^{\prime}\right)=a+b \cdot(1-a)+a \cdot b \cdot(1-a)=a+b-b \cdot a=$ $a \vee b=b$. If $(a, b) \in R$ then by (5) $a C b$ so $R \leqq C$. ( $\left.A, \leqq,^{\prime}\right)$ has property $C$ by (5). If $a, b$ contained in a Boolean subring implies $(a, b) \in$ $R$ for every $a, b \in A$ then $a C b$ implies $(a, b) \in R$ so $C \leqq R$ and hence $R=C$.

3. Weak partial Baer*-semigroups. In this section we continue our study of partial semigroups by enriching their structure through 
the addition of an involution and the Baer property. The main motivation for this section will be found in $\S 5$ where it is shown that the residuated maps on an orthocomplemented poset form a weak partial Baer*-semigroup.

Let $(S, R, \cdot)$ be a partial semigroup. $(S, R, \cdot)$ is a weak involution partial semigroup if there is a partial order relation $\subseteq$ on $S$ and a map*: $S \rightarrow S$ (called an involution) such that $a \subseteq a^{* *}$ and $a^{*} \cdot b^{*} \subseteq$ $(b \cdot a)^{*}$ if these expressions exist. A weak zero 0 is an element in $S$ which satisfies: (i) $(0, a) \in R$ for all $a \in S$ and $0 \cdot a=0$; (ii) if $0 \leqq a$, then $a=0$; (iii) if $a \leqq 0$, then $a^{*}=0$; (iv) if $a \subseteq 0$, then $(a, b),(b, a) \in R$ for all $b \in S$ and $a \cdot b \subseteq 0, b \cdot a \subseteq 0$. Notice a zero is unique if it exists since if $e$ is another zero then $0=e \cdot 0 \subseteq e$ so $e=0$. Notice also that $0^{*}=0$. An element $e \in S$ is a projection if $(e, e) \in R$ and $e=e \cdot e=e^{*}$. A weak involution partial semigroup $S$ is a weak partial Baer*-semigroup if $S$ has a weak zero 0 and if for any $a \in S$ there is a projection $a^{\prime}$ with the following properties:

1. if $\left(a^{\prime}, b\right) \in R$ and $a^{\prime} \cdot b=b$, then $(a, b) \in R$ and $a \cdot b \subseteq 0$;

2. conversely, if $(a, b) \in R$ and $a \cdot b \subseteq 0$, then $\left(b^{*}, a^{\prime}\right) \in R$ and $b^{*} \cdot a^{\prime}=b^{*}$.

Properties (1) and (2) are called the weak Baer properties. This definition of a weak partial Baer*-semigroup generalizes the definition of Gudder and Schelp [6] of a partial Baer*-semigroup which in turn generalizes Foulis's definition [3] of a Baer*-semigroup. In fact a weak partial Baer*-semigroup $\left(S, R, \cdot, \subseteq,{ }^{*},^{\prime}\right)$ is a partial Baer*-semigroup if and only if $\subseteq$ is equality. Thus a partial Baer*-semigroup $\left(S, R, \cdot, *,^{\prime}\right)$ is a partial semigroup $(S, R, \cdot)$ with an involution* satisfying $a=a^{* *},(a \cdot b)^{*}=b^{*} \cdot a^{*}$ if $(a, b) \in R$, a zero 0 satisfying $(0, a) \in$ $R$ with $0 \cdot a=0$ for all $a \in S$ and the property: $(a, b) \in R$ with $a \cdot b=$ 0 if and only if $\left(a^{\prime}, b\right) \in R$ with $a^{\prime} \cdot b=b$. Finally a partial Baer*semigroup $\left(S, R, \cdot, *^{\prime}\right)$ is a Baer*-semigroup if and only if $R=S \times S$. An example of a weak partial Baer*-semigroup that is not a partial Baer*-semigroup is the set of densely defined linear operators on a Hilbert space where $A^{*}$ is the adjoint of the operator $A, A \subseteq B$ means $A$ is a restriction of $B$ and $\cdot$ is composition [17]. An example of a partial Baer*-semigroup that is not a Baer*-semigroup is the set of rectangle matrices $[6,18]$.

For the rest of this section $S$ will denote a weak partial Baer*semigroup $\left(S, R, \cdot, \subseteq, *,^{\prime}\right)$. For convenience we shall omit $\cdot$ and denote $a \cdot b$ by $a b$. Notice since $a^{\prime} a^{\prime}=a^{\prime}$ it follows from (1) that $a a^{\prime} \leqq 0$.

Lemma 3.1. If $a \in S$, then $a^{\prime}$ is unique.

Proof. Suppose $e$ is another projection satisfying (1) and (2). Then since $a a^{\prime} \leqq 0$ we have $a^{\prime} e=a^{\prime}$. Similarly $e a^{\prime}=e$ so $e a^{\prime}=e^{*}=$ 
$\left(e a^{\prime}\right)^{*} \supseteqq a^{\prime} e=a^{\prime}$. Thus $a^{\prime} \subseteq e$ and in a similar way $e \leqq a^{\prime}$ so $e=a^{\prime}$.

Let $P=P(S)$ be the set of projections on $S$. If $e, f \in P$ define $e \leqq f$ if $(e, f) \in R$ and $e f=e$. Notice if $(f, e) \in R$ this would imply that $f e \subseteq e$.

Lemma 3.2. (1) $\leqq$ is a partial order relation. (2) If $e \leqq f$ then $f^{\prime} \leqq e^{\prime}$. (3) If $f \leqq e, e^{\prime}$, then $f=0$. In particular if $e \leqq e^{\prime}$ then $e=0$.

Proof. (1) Clearly $e \leqq e$ for all $e \in P$. Suppose $e \leqq f$ and $f \leqq e$. Then $e=e f \subseteq f$. Similarly $f \leqq e$ so $f=e$. Next suppose $e \leqq f$ and $f \leqq g$. Then $(e, f),(f, g),(e, f g) \in R$. Hence $(e f, g) \in R$ and $e=e f=$ $e(f g)=(e f) g=e g$ and $e \leqq g$. (2) Since $f f^{\prime} \leqq 0$ we have $\left(f, f^{\prime}\right),(e, f)$, $\left(e, f f^{\prime}\right) \in R$. Hence $\left(e f, f^{\prime}\right) \in R$ and $e f^{\prime}=(e f) f^{\prime}=e\left(f f^{\prime}\right) \subseteq 0$. Therefore $f^{\prime} e^{\prime}=f^{\prime}$ so $f^{\prime} \leqq e^{\prime}$. (3) We have $f=f e^{\prime}=(f e) e^{\prime}=f\left(e e^{\prime}\right) \leqq 0$. Hence $f=f^{*}=0$.

We say that a projection $e \in P$ is closed if $e=e^{\prime \prime}$ and denote the closed projections by $P_{c}=P_{c}(S)$.

Theorem 3.3. $P_{c}$ is an orthocomplemented poset.

Proof. $\quad P_{c}$ is partially ordered by Lemma 3.2 (1). If $e \in P_{c}$ then $e=e^{\prime \prime}$ and hence $e^{\prime}=\left(e^{\prime \prime}\right)^{\prime}=\left(e^{\prime}\right)^{\prime \prime}$ so $e^{\prime} \in P_{c}$. If $e \leqq f$ then $f^{\prime} \leqq e^{\prime}$ by Lemma 3.2(2). Since $00=0=0^{*}$ we have $0 \in P$ and since $0 e=0$ for all $e \in P$, we have $0 \leqq e$ for all $e \in P$. Now $0 \leqq 0^{\prime}$ and hence $0^{\prime \prime} \leqq 0^{\prime}$ and $0^{\prime \prime} \leqq\left(0^{\prime \prime}\right)^{\prime}$. By Lemma 3.2(3) $0^{\prime \prime}=0$ and hence $0 \in P_{c}$. If $e \in P_{c}$ and $f \leqq e, e^{\prime}$ then applying Lemma 3.2(3) $f=0$ so $e \wedge e^{\prime}=0$.

One can give examples [6] which show that $P_{c}$ need not be an orthomodular poset.

4. Residuated Mappings. In this section we consider residuated maps on posets. Since our residuated maps need not be defined on the entire poset, our definition is more general than that given by Derderian [2].

Let $(P, \leqq)$ and $(Q, \leqq)$ be posets. If $\phi$ maps a subset of $P$ to $Q$ we denote the domain of $\phi$ by $D(\phi) \cong P$ and the range (image) of $\phi$ by $R(\phi) \cong Q$. A map $\phi: P \supseteqq D(\phi) \rightarrow Q$ is isotone if $a \dot{\phi} \leqq b \dot{\phi}$ whenever $a, b \in D(\phi)$ and $a \leqq b$. An isotone map $\phi: P \supseteqq D(\phi) \rightarrow Q$ is residuated if for every $b \in R(\phi)$ the set $\{a \in D(\phi): a(\phi) \leqq b\}$ has a largest element. Let $S(P, Q)$ be the set of residuated maps from subsets of $P$ to $Q$ and let $S(P)=S(P, P)$. Let $P, Q, R$ be sets and let $\phi: P \supseteqq D(\dot{\phi}) \rightarrow Q, \psi: Q \supseteqq$ $D(\psi) \rightarrow R$. Then the map $\phi \circ \psi: P \supseteqq D(\phi \circ \psi) \rightarrow R$ is defined by $D(\phi \circ \psi)=$ $\{a \in D(\dot{\phi}): a \dot{\phi} \in D(\psi)\}$ and $a \dot{\phi} \circ \psi=(a \dot{\phi}) \psi$ for all $a \in D(\dot{\phi} \circ \psi)$. 
THEOREM 4.1. An isotone map $\phi: P \supseteqq D(\phi) \rightarrow Q$ is residuated if and only if there is an isotone map $\psi: Q \supseteqq D(\psi) \rightarrow P$ such that $R(\phi) \cong$ $D(\psi), R(\psi) \leqq D(\psi)$ with a $\circ \circ \psi \geqq a$ for every $a \in D(\phi)$ and $b \psi \circ \phi \leqq b$ for every $b \in D(\psi)$.

Proof. For sufficiency, if $b \in R(\dot{\phi})$ we claim that $b \psi$ is the largest element of $A=\{a \in D(\dot{\phi}): a \dot{\phi} \leqq b\}$. Indeed if $a \in A$ then $a \dot{\phi} \leqq b$ so $a \leqq$ $(a \phi) \psi \leqq b \psi$. Also $(b \psi) \phi \leqq b$ so $b \psi \in A$. For necessity define $\psi: Q \supseteqq$ $D(\psi) \rightarrow P$ by $D(\psi)=R(\phi)$ and for $b \in D(\psi)$ let $b \psi$ be the largest element in $\{a \in D(\phi): a \phi \leqq b\}$. Note $R(\psi) \subseteq D(\phi)$. If $a, b \in D(\psi)$ and $a \leqq$ $b$ then $\{c \in D(\phi): c \phi \leqq \alpha\} \leqq\{c \in D(\phi): c \phi \leqq b\}$ so $a \psi \leqq b \psi$ and $\psi$ is isotone. If $a \in D(\phi)$ then $(\alpha \phi) \psi$ is the largest element in $\{c \in D(\phi): c \dot{\phi} \leqq a \phi\}$ so $(a \phi) \psi \geqq a$. If $b \in D(\psi)$ then $b \psi \in A$ so $(b \psi) \phi \leqq b$. We can get an even stronger result. Since there is an $a \in D(\phi)$ such that $b=a \dot{\phi}$, we have $(b \psi) \phi=((a \phi) \psi) \phi \geqq a \dot{\phi}=b$. Hence $(b \psi) \dot{\phi}=b$.

A map $\psi$ that satisfies the conditions of Theorem 4.1 is called a residual of $\dot{\phi}$. In general a residuated map may have many residuals. If $\phi: P \supseteqq D(\phi) \rightarrow Q$ and $S \cong D(\phi)$ we denote the restriction of $\phi$ to $S$ by $\left.\phi\right|_{s}$.

Corollary 4.2. Let $\phi \in S(P, Q)$, $\psi$ a residual of $\phi, \phi_{1}=\left.\phi\right|_{R(\psi)}$ and $\psi_{1}=\left.\psi\right|_{R(\phi)}$. Then $a \dot{\phi}_{1} \circ \psi_{1}=a$ for every $a \in R\left(\psi_{1}\right)$ and $a \psi_{1} \circ \phi_{1}=a$ for every $a \in R\left(\phi_{1}\right)$ and hence $R\left(\dot{\phi}_{1}\right)$ and $R\left(\psi_{1}\right)$ are isomorphic.

Proof. Let $a \in R\left(\psi_{1}\right)$. Then by Theorem $4.1 a \phi_{1} \circ \psi_{1}=a \phi_{1} \circ \psi \geqq a$. Now $a=b \psi_{1}$ for some $b \in D\left(\psi_{1}\right)$. Hence $a \phi_{1} \circ \psi_{1}=\left(b \psi_{1} \circ \phi_{1}\right) \psi_{1} \leqq b \psi_{1}=a$. Therefore, $a \phi_{1} \circ \psi_{1}=a$ for all $a \in R\left(\psi_{1}\right)$. Similarly $a \psi_{1} \circ \phi_{1}=a$ for all $a \in R\left(\dot{\phi}_{1}\right)$.

Lemma 4.3. Let $P, Q$ be posets and suppose $Q$ has a first element 0 . If $\dot{\phi} \in S(P, Q)$ and $\psi$ is a residual of $\phi$ with $0 \in D(\psi)$ then for $a \in$ $D(\phi) \alpha \dot{\phi}=0$ if and only if $\alpha \leqq 0 \psi$. Thus if $0 \in D(\phi)$ we have $0 \phi=0$.

Proof. For necessity, $a \leqq a \phi \circ \psi=0 \psi$. For sufficiency, $a \dot{\phi} \leqq$ $0 \dot{\psi} \circ \dot{\phi} \leqq 0$ so $a \dot{\phi}=0$.

Suppose $\phi \in S(P, Q)$ and $\psi \in S(Q, R)$ with residuals $\dot{\phi}_{1}, \psi_{1}$ respectively. Then one might except that $\phi \circ \psi \in R(P, R)$ with residual $\psi_{1} \circ \phi_{1}$ as is the case when $D(\phi)=P, D(\psi)=Q$. However in trying to prove this, one runs into problems with domains. We now prove sufficient conditions for these facts to hold.

TheOREM 4.4. Let $\dot{\phi} \in S(P, Q)$ and $\psi \in S(Q, R)$. If $R(\dot{\phi}) \subseteq D(\psi)$ and $\phi$ has residual $\phi_{1}$ such that $D(\psi) \subseteq D\left(\phi_{1}\right)$ then $\phi \circ$ ' $\in S(P, R)$ with residual $\psi_{1} \circ \phi_{1}$ where $\psi_{1}$ is any residual of $\psi$. 
Proof. Clearly $\phi \circ \psi$ and $\psi_{1} \circ \phi_{1}$ are isotone. Now $R(\phi \circ \psi) \subseteq R(\psi) \subseteq$ $D\left(\psi_{1}\right)=D\left(\psi_{1} \circ \phi_{1}\right)$ since $R\left(\psi_{1}\right) \subseteq D(\psi) \subseteq D\left(\phi_{1}\right)$. Also $R\left(\psi_{1} \circ \phi_{1}\right) \subseteq R\left(\phi_{1}\right) \subseteq$ $D(\phi)=D(\phi \circ \psi)$ since $R(\phi) \subseteq D(\psi)$. If $a \in D(\phi \circ \psi)$ then $(a \phi \circ \psi) \psi_{1} \circ \phi_{1}=$ $\left((a \dot{\phi}) \psi \circ \psi_{1}\right) \phi_{1} \geqq a \phi \circ \phi_{1} \geqq a$ and if $a \in D\left(\psi_{1} \circ \phi_{1}\right)$ then $\left(a \psi_{1} \circ \phi_{1}\right) \phi \circ \psi=$ $\left(\left(a \psi_{1}\right) \phi_{1} \circ \phi\right) \psi \leqq a \psi_{1} \circ \psi \geqq a$.

Theorem 4.5. Let $\phi \in S(P, Q)$ and $\psi \in S(Q, R)$. If $\phi$ and $\psi$ have residuals $\phi_{1}$ and $\psi_{1}$ respectively such that $D\left(\phi_{1}\right) \subseteq D(\psi)$ and $R\left(\psi_{1}\right) \subseteq$ $D\left(\phi_{1}\right)$ then $\phi \circ \psi \in S(P, R)$ with residual $\psi_{1} \circ \phi_{1}$.

Proof. $\quad R(\phi \circ \psi) \subseteq R(\psi) \subseteq D\left(\psi_{1}\right)=D\left(\psi_{1} \circ \phi_{1}\right)$ since $R\left(\psi_{1}\right) \subseteq D\left(\dot{\phi}_{1}\right)$. $R\left(\psi_{1} \circ \phi_{1}\right) \subseteq R\left(\phi_{1}\right) \subseteq D(\phi)=D(\dot{\phi} \circ \psi)$ since $R(\dot{\phi}) \subseteq D\left(\phi_{1}\right) \subseteq D(\psi)$. The rest of the proof is similar to Theorem 4.4.

Corollary 4.6. If $\phi \in S(P, Q), \psi \in S(Q, R)$ and $\phi_{1}$ is a residual of $\phi$ satisfying $D(\psi)=D\left(\phi_{1}\right)$ then $\phi \circ \psi \in S(P, R)$ with residual $\psi_{1} \circ \phi_{1}$ where $\psi_{1}$ is any residual of $\psi$.

Lemma 4.7. If $\phi \in S(P, Q)$ and $\phi_{1}, \phi_{2}$ are residuals of $\phi$ then $\phi_{1}=$ $\dot{\phi}_{2}$ on $D\left(\phi_{1}\right) \cap D\left(\phi_{2}\right)$.

Proof. For $b \in D\left(\phi_{1}\right) \cap D\left(\phi_{2}\right)$ let $a=b \phi_{1}$. Then $a \phi=b \dot{\phi}_{1} \circ \phi \leqq b$. Hence $b \phi_{2} \geqq a \phi \circ \phi_{2} \geqq a=b \phi_{1}$. Similarly $b \phi_{1} \geqq b \phi_{2}$ and hence $b \phi_{1}=b \phi_{2}$ for all $b \in D\left(\dot{\phi}_{1}\right) \cap D\left(\phi_{2}\right)$.

We now show that every residuated map has a unique maximal residual. If $\phi$ and $\psi$ are maps on a set $P, D(\phi) \subseteq D(\psi)$ and $\psi=\dot{\phi}$ on $D(\phi)$ (i.e., $\phi$ is a restriction of $\gamma^{\prime}$ ) we write $\phi \subseteq \psi$.

Theorem 4.8. If $\phi \in S(P, Q)$ then $\phi$ has a largest residual $\phi^{+}$in the sense that if $\psi$ is a residual of $\dot{\phi}$ then $\psi \subseteq \phi^{+}$.

Proof. Let $\Phi$ be the set of all residuals of $\phi$. Then $\Phi$ becomes a poset under the partial order $\subseteq$. Using Zorn's lemma there is a maximal element $\phi^{+} \in \Phi$. We now show that $\phi^{+}$is the only maximal element in $\Phi$. Suppose $\dot{\phi}_{1} \in \Phi$ is another maximal element. Define $\dot{\phi}_{2}$ by $D\left(\phi_{2}\right)=D\left(\phi^{+}\right) \cup D\left(\phi_{1}\right)$ and $a \phi_{2}=a \dot{\phi}^{+}$for $a \in D\left(\phi^{+}\right)$and $a \phi_{2}=a \phi_{1}$ for $a \in D\left(\dot{\phi}_{1}\right)$. Now $\dot{\phi}_{2}$ is well-defined since by Lemma $4.7 \phi_{1}=\dot{\phi}^{+}$on $D\left(\phi^{+}\right) \cap$ $D\left(\phi_{1}\right)$. Then clearly $R(\dot{\phi}) \subseteq D\left(\phi_{2}\right), R\left(\dot{\phi}_{2}\right) \subseteq D(\phi), a \phi^{\circ} \circ \phi_{2} \geqq a$ and $b \dot{\phi}_{2} \circ \phi \leqq b$ for all $a \in D(\phi), b \in D\left(\phi_{2}\right)$. To show $\phi_{2}$ is isotone suppose $a \in D\left(\dot{\phi}^{+}\right), b \in$ $D\left(\phi_{1}\right)$ and $a \leqq b$. Then $a \phi^{+} \circ \phi \leqq a \leqq b$ and hence $a \dot{\phi}^{+} \leqq\left(a \dot{\phi}^{+}\right) \dot{\phi} \circ \phi_{1}=$ $\left(a \phi_{+} \circ \phi\right) \phi_{1} \leqq b \phi_{1}$. Therefore $a \phi_{2}=a \phi^{+} \leqq b \dot{\phi}_{1}=b \phi_{2}$. We thus see that $\phi_{2}$ is a residual of $\phi$ and by maximality $\dot{\phi}_{2}=\phi_{1}=\phi^{+}$.

Let us now consider $S(P)$ for a poset $P$. Define the relation $R \subseteq$ 
$S(P) \times S(P)$ by $(\phi, \psi) \in R$ if $\phi \circ \psi \in S(P)$. It is then easy to see that $(S(P), R, \circ)$ is a partial semigroup. In the next section we show that if $P$ is an orthocomplemented poset then the above partial semigroup has a much richer structure.

5. Adjoint mappings on orthocomplemented posets. We now specialize further and assume in the sequel that $P$ is an ortho-complemented poset. We will also only consider $\phi \in S(P)$ which satisfy $\{0,1\} \subseteq D(\phi)$. If $\phi \in S(P)$ and $\phi^{+}$is its largest residual we define $\phi^{*}: P \supseteqq D\left(\phi^{*}\right) \rightarrow P$ by $D\left(\phi^{*}\right)=D\left(\phi^{+}\right)^{\prime}=\left\{a^{\prime}: a \in D\left(\phi^{+}\right)\right\}$and $a \phi^{*}=\left(a^{\prime} \phi^{+}\right)^{\prime}$.

LEMMA 5.1. $\phi^{*}$ is an isotone map with the following properties:

(i ) $R(\phi)^{\prime} \subseteq D\left(\phi^{*}\right), R\left(\phi^{*}\right)^{\prime} \subseteq D(\phi)$;

(ii) $(a)^{\prime} \phi^{*} \leqq a^{\prime}$ and $\left(b \phi^{*}\right)^{\prime} \phi \leqq b^{\prime}$ for all $a \in D(\phi), b \in D\left(\phi^{*}\right)$;

(iii) If $\phi_{1}$ is an isotone map with properties (i) and (ii) then $\phi_{1} \subseteq \phi^{*}$.

Proof. To show $\phi^{*}$ is isotone suppose $a, b \in D\left(\phi^{*}\right)$ and $a \leqq b$. Then $b^{\prime} \leqq a^{\prime}$ so $b^{\prime} \phi^{+} \leqq a^{\prime} \phi^{+}$and hence $a \phi^{*}=\left(a^{\prime} \phi^{+}\right)^{\prime} \leqq\left(b^{\prime} \phi^{+}\right)^{\prime}=b \phi^{*}$. (i) Since $R(\phi) \subseteq D\left(\phi^{+}\right)$we have $R(\phi)^{\prime} \leqq D\left(\phi^{+}\right)^{\prime}=D\left(\phi^{*}\right)$ and also $R\left(\phi^{*}\right)^{\prime}=R\left(\phi^{+}\right) \subseteq D(\phi)$. (ii) If $a \in D(\phi)$, then $(a \phi)^{\prime} \phi^{*}=\left(a \phi^{\prime} \circ \phi^{+}\right)^{\prime} \leqq a^{\prime}$ and if $b \in D\left(\phi^{*}\right)$, then $\left(b \phi^{*}\right)^{\prime} \phi=b^{\prime} \dot{\phi}^{+} \circ \phi \leqq b^{\prime}$. (iii) follows from Theorem 4.8 .

Any isotone map that satisfies ( $\mathrm{i}$ ) and (ii) Lemma 5.1 is called an adjoint of $\phi$ and the map $\phi^{*}$ is called the maximal adjoint of $\phi$. Notice that any map with an adjoint is residuated.

We now show that $S(P)$ can be made into a weak involution partial semigroup with a weak zero. The map $a 0=0$ for all $a \in P$ will be the weak zero. Define the relation $R=\{(\phi, \psi) \in S(P) \times S(P)$ : $\psi \cong 0$, or if $\psi \varsubsetneqq 0, R(\phi) \subseteq D(\psi)$ and $\phi \circ \psi \in S(P)\}$. If $(\phi, \psi) \in R$ with $\psi \nsubseteq 0$ define $\phi \cdot \psi=\phi \circ \psi$ and if $(\phi, \psi) \in R$ with $\psi \leqq 0$ define $D(\dot{\phi} \cdot \psi)=$ $D(\phi)$ and $\phi \cdot \psi=0$ on $D(\phi)$. For convenience we use the notation $\phi \psi=\phi \cdot \psi$.

Lemma 5.2. $(S(P), R, \cdot, \subseteq, *)$ is a weak involution partial semigroup with a weak zero.

Proof. We leave it to the reader to check that $(S(P), R, \cdot)$ is a partial simigroup and that 0 satisfied the properties of a weak zero. If $\phi \in S(P)$ then $\phi^{*} \in S(P)$ and since $\phi$ is an adjoint of $\phi^{*}$ we have $\phi \leqq \phi^{* *}$. Now suppose $(\phi, \psi),\left(\psi^{*}, \phi^{*}\right) \in R$ and $\psi^{\prime}, \phi^{*} \nsubseteq 0$. Then for all $e \in D(\dot{\phi} \psi)$ we have $(e \phi \psi)^{\prime}\left(\psi^{*} \phi^{*}\right) \leqq(e \phi)^{\prime} \phi^{*} \leqq e^{\prime}$. Similarly for all $f \in$ $D\left(\psi^{*} \phi^{*}\right)$ we have $\left(f \psi^{*} \phi^{*}\right)^{\prime}(\phi \psi) \leqq\left(f \psi^{*}\right)^{\prime} \psi \leqq f^{\prime}$. If $\psi \leqq 0$, then $\phi \psi \subseteq 0$, so, for $f \in D\left(\psi^{*} \phi^{*}\right)$ we have $\left(f \psi^{*} \phi^{*}\right)^{\prime} \phi \psi=0 \leqq f$. Also, if $\psi \leqq 0$, then 
$\psi^{*}=0$, so $\psi^{*} \phi^{*} \leqq 0$ and, for $e \in D(\phi \psi)$, we have $(e \phi \psi)^{\prime} \psi^{*} \phi^{*}=0 \leqq e^{\prime}$. If $\phi^{*} \subseteq 0$ then $\phi^{* *}=0$ and hence $\phi \subseteq 0$ and we get the same results as in the previous sentence. We thus have that $\psi^{*} \phi^{*}$ is an adjoint of $\phi \psi^{\prime}$ and so $\psi^{*} \phi^{*} \subseteq(\phi \psi)^{*}$.

Lemma 5.3. (1) If $\phi \in S(P)$ and $a \in D(\phi)$ then $\alpha \dot{\rho}=0$ if and only if $a \leqq\left(1 \phi^{*}\right)^{\prime}$. (2) Suppose $\phi, \psi \in S(P)$ and $(\phi, \psi) \in R$. Then $\phi \psi \leqq 0$ if and only if $1 \phi \leqq\left(1 \psi^{*}\right)^{\prime}$.

Proof. (1) follows from Lemma 4.3. (2) If $1 \phi \leqq\left(1 \psi^{*}\right)^{\prime}$ then

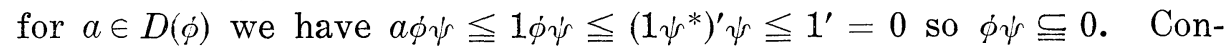
versely, if $\phi \psi \subseteq 0$ then $(1 \phi) \psi=0$ so $1 \phi \leqq\left(1 \psi^{*}\right)^{\prime}$ by part (1).

Gudder and Schelp [6] have shown that any orthomodular poset $P$ can be coordinatized by a partial Baer*-semigroup $S$ in the sense that $P$ is isomorphic to the poset of closed projections in $S$. (In fact a stronger result is given in [6] where it is shown that $S$ is an $(O M)$ partial Baer*-semigroup; however, we shall not consider $(O M)$ partial Baer*-semigroups here.) However, the coordinatization in [6] is very coarse (it is minimal in a certain sense) and for that reason may not reflect some of the important properties of $P$. We now show that $S(P)$ gives a coordinatization of $P$ in a weak partial Baer*-semigroup. This is closer to the coordinatization of an orthomodular lattice given by Foulis [3].

We now assume that $P$ is an orthomodular poset. For $a \in P$ define $\dot{\phi}_{a}$ by $D\left(\dot{\phi}_{a}\right)=[0, a] \cup\left[0, a^{\prime}\right] \cup[a, 1] \cup\left[a^{\prime}, 1\right]$ and if $e \in D\left(\dot{\phi}_{a}\right)$ then $e \dot{\phi}_{a}=$ $e \wedge a$. Notice if $e \in D\left(\phi_{a}\right)$ then $e C a$ so $e \wedge a$ exists. Also if $b \leqq a$ then $b \dot{\phi}_{a}=b$ and hence $\phi_{a} \phi_{a}=\phi_{a}$. Notice that $\phi_{a}$ is isotone. Furthermore $R\left(\dot{\phi}_{a}\right)^{\prime} \leqq D\left(\dot{\phi}_{a}\right)$ and for $e \in D\left(\dot{\phi}_{a}\right)$ we have $\left(e \phi_{a}\right)^{\prime} \dot{\phi}_{a}=\left(e^{\prime} \vee a^{\prime}\right) \wedge a=$ $e^{\prime} \wedge a \leqq e^{\prime}$. Thus $\phi_{a} \in S(P)$ and $\phi_{a} \subseteq \phi_{a}^{*}$. For $\phi \in S(P)$ let $a=\left(1 \phi^{\prime}\right)^{\prime}$ and define $\phi^{\prime}=\phi_{a}^{*}$.

Theorem 5.4. If $P$ is an orthomodular poset then $\left(S(P), R, \cdot, \subseteq, *,{ }^{\prime}\right)$ is a weak partial Baer*-semigroup and $a \rightarrow \phi_{a}^{*}$ is an isomorphism of $P$ onto $P_{c}(S(P))$.

Proof. Let $\phi \in S(P)$ and $a=(1 \phi)^{\prime}$. Then for $e \in D(\phi), e \phi \leqq 1 \phi=$ $a^{\prime}$ so $R(\dot{\phi}) \subseteq D\left(\dot{\phi}_{a}\right)$ and $e \dot{\phi} \dot{\phi}_{a}=e \dot{\phi} \wedge a=0$. Thus $\dot{\phi} \dot{\phi}_{a} \cong 0$ and since $\left(\phi, \phi_{a}^{*}\right) \in R$ we have $\phi \phi_{a}^{*} \subseteq 0$. Suppose $\psi \in S(P)$ and $\phi \dot{\phi} \fallingdotseq 0$. By Lemma 5.3(2) $1 \gamma^{*} \leqq a$ so for all $e \in D\left(\psi^{*}\right), e \psi^{*} \leqq a$, and in particular $e \phi_{a}^{*} \leqq$ $a$ for $e \in D\left(\phi_{a}^{*}\right)$. For $e \in D\left(\psi^{*}\right), e \psi^{*} \phi_{a}^{*}=e \psi^{*} \phi_{a}=e \psi^{*}$. Therefore $\left(\psi^{*}, \phi^{\prime}\right) \in$ $R$ and $\psi^{*} \dot{\phi}^{\prime}=\psi^{*} \dot{\phi}_{a}^{*}=\psi^{*}$. Now suppose $\left(\phi^{\prime}, \psi\right) \in R$ and $\phi^{\prime} \psi^{\prime}=\psi^{\prime}$. Since $R(\phi) \subseteq D\left(\phi_{a}\right) \subseteq D\left(\phi^{\prime}\right)$ we have $(\phi, \psi)=\left(\phi, \phi^{\prime} \psi\right) \in R$ and $\phi^{\prime} \psi=\phi^{\prime}\left(\phi^{\prime} \psi\right)=$ $\left(\phi \phi_{a}^{*}\right) \psi \subseteq 0$. We now show that $\phi_{a}^{*}$ is a projection for all $a \in L$. For $e \in D\left(\phi_{a}^{*}\right)$, since $e \phi_{a}^{*} \leqq a$, we have $\left(e \dot{\phi}_{a}^{*}\right) \phi_{a}^{*}=\left(e \phi_{a}^{*}\right) \phi_{a}=e \phi_{a}^{*}$. We also 
have $R\left(\phi_{a}^{*}\right)^{\prime} \subseteq\left[a^{\prime}, 1\right] \subseteq D\left(\phi_{a}\right) \subseteq D\left(\phi_{a}^{*}\right)$ and $\left(e \phi_{a}^{*}\right)^{\prime} \phi_{a}^{*}=\left(e \phi_{a}^{*}\right)^{\prime} \phi_{a} \leqq e^{\prime}$. Thus $\phi_{a}^{*}$ is an adjoint of itself. Suppose $\psi$ is an adjoint of $\phi_{a}^{*}$ and $\phi_{a}^{*} \subseteq \psi$. We now show that $\psi$ is an adjoint of itself. Indeed, $R(\psi)^{\prime} \subseteq D\left(\phi_{a}^{*}\right) \subseteq$ $D(\psi)$ and for $e \in D(\psi),(e \psi)^{\prime} \psi=\left(e \psi^{\prime} \phi_{a}^{*} \leqq e^{\prime}\right.$. Thus $\psi \subseteq \psi^{*}$. We next show that $\psi$ and $\phi_{a}$ are mutual adjoints. Since $\phi \psi=\phi \phi_{a} \subseteq 0$ we have $e \psi^{*} \leqq a$. Hence $R(\psi)^{\prime} \subseteq R\left(\psi^{*}\right)^{\prime} \leqq D\left(\phi_{a}\right)$ and also $R\left(\phi_{a}\right)^{\prime} \leqq R\left(\phi_{a}^{*}\right) \subseteq D(\psi)$. Now $\left(e \phi_{a}\right)^{\prime} \psi=\left(e \phi_{a}\right)^{\prime} \phi_{a}^{*} \leqq e^{\prime}$ and $\left(f \psi^{\prime}\right)^{\prime} \phi_{a}=(f \psi)^{\prime} \phi_{a}^{*} \leqq f^{\prime}$ for all $e \in D\left(\phi_{a}\right)$ and $f \in D(\psi)$. Thus $\psi$ and $\phi_{a}$ are mutual adjoints, so $\psi \subseteq \phi_{a}^{*}$ and hence $\psi=\phi_{a}^{*}$. Thus $\phi_{a}^{*}$ is a maximal adjoint of itself and we have $\dot{\phi}_{a}^{* *}=\phi_{a}^{*}$. Hence $\phi_{a}^{*}$ is a projection for any $a \in P$. We have thus shown that $\left(S(P), R, \cdot, \subseteq,^{*},{ }^{\prime}\right)$ is a weak partial Baer*-semigroup. Now $\left(\phi_{a}^{*}\right)^{\prime}=\phi_{a}^{* \prime}$ and hence $\left(\phi_{a}^{*}\right)^{\prime \prime}=\phi_{a^{\prime \prime}}^{*}=\phi_{a}^{*}$ which shows that $\phi_{a}^{*}$ is closed. To show that $a \rightarrow \phi_{a}^{*}$ is onto, let $\phi \in P_{c}(S(P))$. Then $\phi^{\prime}=\phi_{a}^{*}$ where $a=(1 \phi)^{\prime}$. Hence $\phi=\phi^{\prime \prime}=\phi_{a}^{* \prime}$. To show $a \rightarrow \phi_{a}^{*}$ is one-one suppose $\phi_{a}^{*}=\phi_{b}^{*}$. Then $a=1 \dot{\phi}_{a}=1 \phi_{a}^{*}=1 \phi_{b}^{*}=1 \dot{\phi}_{b}=b$. If $a, b \in P$ and $a \leqq b$ then $e \phi_{a}^{*} \leqq a$ and we have $e \dot{\phi}_{a}^{*} \dot{\phi}_{b}^{*}=e \phi_{a}^{*} \phi_{b}=e \dot{\phi}_{a}^{*}$ and hence $\dot{\phi}_{a}^{*} \leqq$ $\dot{\phi}_{b}^{*}$. Conversely, if $\left(\phi_{a}^{*}, \phi_{b}^{*}\right) \in R$ and $\phi_{a}^{*} \phi_{b}^{*}=\phi_{a}^{*}$ then $b \geqq a \phi_{b}^{*}=\left(1 \dot{\phi}_{a}^{*}\right) \dot{\phi}_{b}^{*}=$ $1 \phi_{a}^{*}=a$.

6. Operations in axiomatic quantum mechanics. In axiomatic quantum mechanics, the "logic" for a quantum mechanical system is usually taken to be an orthomodular poset (or lattice, for generality we will use posets) $P[7,10,13,19]$. A state on $P$ is a map $m$ from $P$ to the real unit interval $[0,1]$ that satisfies $1 m=1$ and $(\alpha \vee b) m=$ $a m+b m$ if $a$ and $b$ are disjoint. ( $P$ is usually taken to be countably orthocomplete and the states countably additive, but we will not need to assume these here.) Let $M$ denote the set of states on $P$. We say that $M$ is strongly order determining if whenever $\{m \in M: a m=1\} \subseteq$ $\{m \in M: b m=1\}$ then $a \leqq b$, for $a, b \in P$. If $P$ is an orthomodular poset whose set of states $M$ is strongly order determining we call $(P, M)$ a quantum logic.

In the sequel $(P, M)$ will always denote a quantum logic. If $a \in$ $P$, a map $\Omega_{a}: M \supseteqq D\left(\Omega_{a}\right) \rightarrow M$ is a primitive operation (corresponding to $a$ ) if

1. $D\left(\Omega_{a}\right)=\{m \in M: a m \neq 0\}$;

2. if $a m=1$, then $m \Omega_{a}=m$;

3. if $a C b$ and $m \in D\left(\Omega_{a}\right)$ then $b\left(m \Omega_{a}\right)=(a \wedge b) m / a m$.

A primitive operation is physically a conditioning procedure that gives the resulting state $m \Omega_{a}$ after measurement of the quantum proposition a if the initial state is $m$. Our definition is a generalization of Pool's [14] original definition of a primitive operation. Pool gives the following two additional requirements that we shall not postulate (although we will postulate a weakened form of (5)):

4. if $\Omega_{a_{1}}, \cdots, \Omega_{a_{n}}$ are any finite number of primitive operations 
there is an $a \in P$ such that $C D\left(\Omega_{a_{1}} \circ \ldots \circ \Omega_{a_{n}}\right)=\{m \in M: a m=1\}$ (where $C D$ denotes the set-theoretic complement of a set $D$ ).

5 . If $\Omega_{a_{1}} \circ \cdots \circ \Omega_{a_{n}}=\Omega_{b_{1}} \circ \cdots \circ \Omega_{b_{r}}$ then $\Omega_{a_{n}} \circ \cdots \circ \Omega_{a_{1}}=\Omega_{b_{r}} \circ \cdots \circ \Omega_{b_{1}}$. These last two conditions are hard to justify physically and it is our purpose to show that they need not be postulated (except for the weakened form of (5) mentioned above) if one is willing to use partial Baer*-semigroups instead of Baer*-semigroups. Specifically Pool has shown [14] that his primitive operations generate a Baer*-semigroup that coordinatizes $P$. We will show that our weaker primitive operations generate a partial Baer*-semigroup that coordinatizes $P$. (Another difference that should be mentioned is that Pool assumes $P$ is a lattice while we do not.)

Let $B \cong P$ be a sub-orthomodular poset and let $b$ be in the center $Z(B)$ of $B$ (i.e., $b C a$ for all $a \in B$ ). If $m \in M, b m \neq 0$ define $m_{b}: B \rightarrow$ $[0,1]$ by $a m_{b}=(a \wedge b) m / b m$. We call $m_{b}$ a conditional state on $B$. We call $B$ conditional if every conditional state on $B$ has an extension to an element of $M$. We say that $(P, M)$ is conditional if every sub-orthomodular poset of $P$ is conditional. This concept was introduced by the author in [8] where examples are given.

Lemma 6.1. $(P, M)$ is conditional if and only if corresponding to every $a \in P$ there is a primitive operation $\Omega_{a}$.

Proof. For sufficiency let $B$ be a sub-orthomodular poset of $P$, $m \in M, b \in Z(B), b m \neq 0$ and $m_{b}$ a conditional state on $B$. Then $m \Omega_{b}$ is an extension of $m_{b}$ to an element of $M$. For necessity let $a \in P$ and define $D\left(Q_{a}\right)=\{m \in M: a m \neq 0\}$. If $a m=1$ define $m \Omega_{a}=m$. Now suppose $0<a m<1$. Let $B=\{b \in P: b C a\}$. Then $B$ is a sub-orthomodular poset of $P$ and hence for any $m \in M$ with $a m \neq 0 m_{a}$ is a conditional state on $B$. Hence $m_{a}$ has an extension $\hat{m}_{a}$ to $M$ and define $m \Omega_{a}=\hat{m}_{a}$.

In the sequel $(P, M)$ will be a conditional quantum logic. Let $\Sigma$ be the collection of finite compositions of primitive operations $\Omega_{a_{1}} \circ \cdots \circ \Omega_{a_{n}}$ that satisfy:

(i) there are elements $a, b \in P$ such that $C D\left(\Omega_{a_{1}} \circ \cdots \circ \Omega_{a_{n}}\right)=$ $\{m \in M: a m=1\}$ and $C D\left(\Omega_{a_{n}} \circ \cdots \circ \Omega_{a_{1}}\right)=\{m \in M: b m=1\}$;

(ii) $\Omega_{a_{1}} \circ \cdots \circ \Omega_{a_{n}}=\Omega_{b_{1}} \circ \cdots \circ \Omega_{b_{r}}$ for some $b_{1}, \cdots, b_{r} \in P$ if and only if $\Omega_{a_{n}} \circ \cdots \circ \Omega_{a_{1}}=\Omega_{b_{r}} \circ \cdots \circ \Omega_{b_{1}}$.

Lemma 6.2. The set of primitive operations is a subset of $\Sigma$ if and only if the following condition holds: (5') if $\Omega_{a_{1}} \circ \cdots \circ \Omega_{a_{n}}=\Omega_{a}$ then $\Omega_{a_{n}} \circ \cdots \circ \Omega_{a_{1}}=\Omega_{a}$, for any $a, a_{1}, \cdots, a_{n} \in P$.

Proof. Necessity follows from (ii) above. For sufficiency, 
implies that (ii) holds for primitive operations. Now given a primitive operation $\Omega_{a}$ we have $C D\left(\Omega_{a}\right)=\{m \in M: a m=0\}=\left\{m \in M: a^{\prime} m=1\right\}$ so (i) holds for primitive operations.

Notice that $\left(5^{\prime}\right)$ is a weaker postulate than (5) and it turns out to be easier to justify physically.

We define the relation $R \subseteq \Sigma \times \Sigma$ by $(f, g) \in R$ if $f \circ g \in \Sigma$. Thus $(\Sigma, R, \circ)$ is partial semigroup. If $f=\Omega_{a_{1}} \circ \cdots \circ \Omega_{a_{n}} \in \Sigma$ define $f^{*}=$ $\Omega_{a_{n}} \circ \cdots \circ Q_{a_{1}} \in \Sigma$. It is easy to see that $*$ is an involution and is in fact the unique involution on $\Sigma$ such that $\Omega_{a}=\Omega_{a}^{*}$ for all $a \in P$.

THEOREM 6.3. If $(P, M)$ is a conditional quantum logic satisfying $\left(5^{\prime}\right)$ then $\Sigma$ is a partial Baer*-semigroup which coordinatizes $P$.

Proof. We already have that $(\Sigma, R, \circ)$ is a partial involution semigroup. It is easy to see that $\Omega_{0} \equiv 0$ is the zero for $\Sigma$. Now since using (3) $a\left(m \Omega_{a}\right)=1$ we have using (2) that $m \Omega_{a} \circ \Omega_{a}=m \Omega_{a}$ for all $m \in\left(\Omega_{a}\right)$ and hence the primitive operations are projections in $\Sigma$. Now for $f \in \Sigma$ let $a \in P$ be the unique element that satisfies $C D(f)=$ $\{m \in M: a m=1\}$ and let $f^{\prime}=\Omega_{a}$. The proof that $\left(\Sigma, R, \circ, *,^{\prime}\right)$ is a partial Baer*-semigroup and that the map $a \rightarrow \Omega_{a}$ gives a coordinatization follows along similar lines to the proof of Theorem II 3 [14].

\section{REFERENCES}

1. G. Birkhoff, Lattice Theorey, Amer. Math. Soc. Colloq. Publ. Vol. XXV Providence, R. I. (1967).

2. J. Dedérian, Residuated mappings, Pactific J. Math., 20 (1967), 35-43.

3. D. Foulis, Baer*-Semigroups, Proc. Amer. Math. Soc., 11 (1960), 648-654.

4. G. Grätzer and G. Wenzel, On the concept of congruence relation in partial algebras, Math. Scand., 20 (1967), 275-280.

5. G. Grätzer and E. Schmidt, Characterization of congruence lattices of abstract algebras, Acta Sci. Math., (Szeged) 24 (1963), 34-59.

6. S. Gudder and R. Schelp, Coordinatization of orthocomplemented and orthomodular posets, Proc. Amer. Math. Soc., 25 (1970), 229-237.

7. S. Gudder, Axiomatic quantum mechanics and generalized probability theory, in A. Bharucha-Reid (editor) Probabilistic Methods in Applied Mathematics Vol. II, Academic Press, New York (1970).

8. - On the quantum logic approach to quantum mechanics, Commum. Math. Phys., 12 (1969), 1-15.

9. - Spectral methods for a generalized propability theory, Trans. Amer. Math. Soc., 119 (1965), 428-442.

10. J. Jauch, Foundations of Quantum Mechanics, Addison-Wesley, Reading, Mass. (1968).

11. S. Kochen and E. Specker, Logical structures arising in quantum theory, Symposium on the Theory of Models, North-Holland Publ. Co., Amsterdam (1965) 177-189.

12. - The problem of hidden variables in quantum mechanics, J. Math. Mech., 17 (1967), 59-87.

13. G. Mackey, Mathematical Foundations of Quantum Mechanics, Benjamin, New York (1963). 
14. J. Pool, Baer*-semigroups and the logic of quantum mechanics, Commun. Math. Phys., 9 (1968), 118-141.

15. - Simultaneous observables and the logic of quantum mechanics, Tech. Report State Univ., Iowa (1963).

16. A. Ramsey, A theorem on two commuting observables, J. Math. Mech., 15 (1966), 227-234.

17. F. Riesz and B. Sz. Nagy, Functional Analysis, Frederick Ungar Publ. Co., New York (1955).

18. R. Schelp, Partial Baer*-semigroups and partial Baer semigroups, Dissertation, Kansas State Univ. Manhattan, Kansas.

19. V. Varadarajan, Geometry of Quantum Theory Vol. I, Van Nostrand, Princeton, N. J. (1968).

Received September 20, 1970 and in revised form October 21, 1971.

UNIVERSITY OF DENVER 